\title{
MS44-04 | Structure Study OF A Disordered Zeolite by CRED ANd HRTEM
}

Zhao, Jingjing (Stockholm University, Stockholm, SWE)

Structure study of a disordered zeolite by electron crystallography

Intergrowth and stacking disorders occur very often in synthetic zeolites, making their structural determination very challenging. Electron crystallography is a powerful method, which enables us to solve disordered structures by combining reciprocal (3D electron diffraction) and direct space (high-resolution transmission electron microscopy, HRTEM) methods. Continuous rotation electron diffraction (cRED) is an effective and high-throughput method for structure determination of micron- to nano-sized single crystals [1-3]. HRTEM images can give us local information in direct space, which is crucial to study disordered materials [4,5]. In this work, a new and disordered aluminosilicate zeolite was studied by the combination of cRED and HRTEM. cRED data were used to determine the average structure of this zeolite, whereas HRTEM images were used to study the disorder types.

[1] Nannenga, B. L. et al. Nature Methods 11, 927 (2014).

[2] Wang, Y. et al. Journal of Applied Crystallography 51, 1094-1101 (2018).

[3] Cichocka, M. O. et al. Journal of Applied Crystallography 51, 1652-1661 (2018).

[4] Willhammar, T. et al. Nature Chemistry 4, 188-194 (2012).

[5] Cichocka, M. O. et al. Crystal Growth \& Design 18, 2441-2451 (2018). 